\title{
EDITORIAL
}

\section{Tuberculosis in evolution}

\author{
György Pálfi a, *, Olivier Dutour ${ }^{\mathrm{b}, \mathrm{c}}$, Pascale Perrin ${ }^{\mathrm{d}}$, Christophe Sola ${ }^{\mathrm{e}}$, Albert Zink ${ }^{\mathrm{f}}$ \\ a Department of Biological Anthropology, University of Szeged, Szeged, Hungary \\ b Laboratoire d'anthropologie biologique Paul Broca, École Pratique des Hautes Études, UMR 5199 PACEA, Université de Bordeaux, Pessac, France \\ ${ }^{\mathrm{c}}$ Department of Anthropology, University of Western Ontario, Canada \\ ${ }^{\mathrm{d}}$ Unité de Recherche MIVEGEC - UR 224/IRD - UMR 5290/CNRS - UM1 - Université Montpellier 2, Montpellier, France \\ e Institut de Biologie Intégrative de la Cellule (I2BC), CEA-CNRS-UPS, UMR9198, Bât 400, F-91400 Orsay, France \\ ${ }^{\mathrm{f}}$ Institute for Mummies and the Iceman, EURAC Research, Bolzano, Italy
}

At the beginning of the 1980 s, about a hundred years after Robert Koch had discovered the pathogen of tuberculosis (TB) in 1882, many still believed that TB, like smallpox, could be eliminated by the end of the millennium from the group of infectious diseases afflicting humanity. This optimistic approach was supported by the spectacular $20^{\text {th }}$-century results in the prevention, diagnostics and therapy of the disease. Sadly, these predictions did not come true. Instead of being eradicated, TB re-emerged at the end of the 20th century to become a global health threat for the new millennium.

According to the latest WHO's data, TB remains one of the world's deadliest contagious diseases despite slowly declining each year. In 2013, an estimated 9 million people developed TB and 1.5 million died from the disease, 360,000 of whom were HIV-positive. A global $3.5 \%$ multi-drug resistance rate was observed. Of the estimated 9 million TB cases, more than half (56\%) were in the South-East Asia and Western Pacific regions. A further one quarter were in the African region, which also had the highest rates of cases and deaths relative to population. India and China alone accounted for $24 \%$ and $11 \%$ of total cases respectively [2].

Tuberculosis remains a major global health problem worldwide. Despite the advent of antibiotics which have widely reduced mortality, antibiotic-resistant strains have now emerged, with multi-resistant strains raising therapeutic problems reminiscent of pre-antibiotherapy clinical situations dating from the turn of the 19th and 20th centuries. This reminder of history prompts us to increase our knowledge both of the origin and evolutionary history of TB and of the factors promoting the emergence and spread of TB strains in human populations worldwide.

Fifteen years after the first multidisciplinary TB meeting ICEPT ("International Congress on The Evolution and Paleoepidemiology of Tuberculosis”; September 1997, Budapest-Szeged, Hungary - see more details in [1]), a further TB Evolution Meeting (ICEPT2) was held in Szeged, Hungary between the 22nd and 25th of March 2012 to gather an updated multidisciplinary synthesis of the

\footnotetext{
* Corresponding author.

E-mail address: palfigy@bio.u-szeged.hu (G. Pálfi).
}

evolutionary pattern of TB. The recent progresses in the knowledge of TB evolutionary biology and the spectacular results produced by the new interdisciplinary collaborations initiated during the first ICEPT demanded a new synthesis of these topics. Several important questions were addressed during ICEPT2, among them: what is the oldest evidence of this infection on human and animal remains? When did specific mutation(s) of the modern strains arise? What was the relative importance of the different pathogenic species of the Mycobacterium genus among past populations? Can we reconstruct a consensual phylogeny of the Mycobacterium genus? How can paleopathology and paleomicrobiology contribute to the research on TB evolution? What are the main strategies to stop or to moderate the re-emergence of TB? How can molecular phylogenetics contribute to these fights? The most up-to-date answers to these questions are providing in this Special Issue of Tuberculosis, which gathers the results of the 2012 ICEPT2 TB Evolution Meeting.

When choosing the dates of the 2012 TB Evolution Meeting, it was decided to join the Albert Szent-Györgyi's Nobel Prize Award Anniversary Conference Series (23 $3^{\text {rd }}-25$ th March 2012, University of Szeged, Szeged, Hungary) and the International TB day (24th March 2012), corresponding to the $130^{\text {th }}$ anniversary of Robert Koch's discovery. This Conference Series therefore also benefitted from the presence of nine Nobel Prize winners.

The first chapter of this special issue is dedicated to the past of TB - and TB in the Past. It is currently usually assumed that most major infectious diseases could have only emerged within the last ten thousand years, following the rise of agriculture and domestication and the growth of human populations during the Neolithic period. Human TB could be an environmental (zoonotic) disease, e.g. derived from animal TB or may have developed gradually by coevolution, specialization and adaptation towards pathogenicity and virulence of human-adapted bacteria. In this special issue, analysis of skeletons coming from the early Neolithic site of Dja'de el Mughara (11th millenium BP) can now reveal that human tuberculosis predated domestication in ancient Syria (Baker et al.), occurring at a site showing only the earliest signs of wild aurochs (Bos primigenius) domestication. Interestingly, archaeological signs of settlement during the 9th millennium BC are restricted to the 
same region. Archaeological and paleoanthropological data available in this area provide adequate material to test the early presence of Mtb complex pathogens. Few studies have been completed on human and animal remains, so the opportunity to test the hypothesis of a co-evolution rather than a direct transmission from bovines to humans is not yet possible. However, we cannot exclude that TB may also been contracted from wild or feral animals (Minnikin et al.). In that case, phylogeographical specificity of ancient wildlife and various food regimens may have played a role. With the assumption of a human Mycobacterium tuberculosis ancestor, the question of TB identification in Upper Paleolithic modern humans if not among Neanderthals can now be asked.

The vast majority of infectious diseases only affect the soft tissues of the body. In the case of TB, only a small proportion of patients (3-5\%) develops bone stigma. It is therefore a great challenge to analyze and detect specific bone lesions to target samples of interest. The remarkable bone changes indicative of a longstanding chronic disease (Pott's disease) would have been unlikely to develop until the infection was less virulent. Until now, paleopathology was limited to the identification of this small proportion of individuals carrying such specific lesions, having suffered from TB for an extensive period of time. Fortunately, mycobacteria are suitable targets for paleopathological and biomolecular studies because of their waxy, hydrophobic and lipidrich cell wall (Daffé) that seems to help to protect DNA against destruction and degradation. New approaches combining morphological studies (minor osteological lesions, microscopic changes) with biochemical and molecular analyses of biological traces in bones (specific lipid biomarkers, proteins, DNA) provide promising results with the detection and identification of ancient TB infection (e.g. Donoghue et al.; Hershkovitz et al.; Masson et al.; Molnár et al.; Pálfi et al.; Santos; Schmidt-Schultz and Schultz; Schultz and Schmidt-Schultz).

Paleopathological processes of TB infection can also be better understood by 3D imaging (Coqueugniot et al.). Information is now also available on TB strains which had infected ancient human populations in different geographic regions. Furthermore, new methods can now address paleoepidemiological questions about the prevalence and distribution of infection diseases in past populations (Blondiaux and Naji; Eddy; Teschler-Nicola et al.). New studies using the molecular analysis identification of $M$. tuberculosis in prehistoric and historic populations between $5000 \mathrm{BC}$ and 1800 $\mathrm{AD}$ give a more complete view on the infection rates in ancient times (Masson et al.; Lösch et al.; Pósa et al.; Szikossy et al.). They also provide information about the molecular evolution and the phylogenetic relationships of infectious diseases through the identification of possible TB strains no longer found today as well as the comparative analysis of modern and ancient microbial sequences (Roberts; Spigelman et al.).

More than 2000 modern MTB genomes have been sequenced to date, affording a more accurate reconstruction and calibration of the evolutionary scheme of $M$. tuberculosis helping to evaluate the distribution and prevalence of ancient strains occurring both in ancient and modern populations. It must be stressed that such studies do not necessarily demonstrate the proportion of individuals that died from TB, but rather the proportion that had contracted TB at some stages of their lives. Knowledge of changing prevalence in the past helps epidemiologists to understand the effect of environmental and socio-cultural factors affecting infectious diseases at a time before antibiotics and other modern interventions (Matos and Santos; Roberts and Bernard).

Ancient biomolecular analyses, including new sequencing technologies (next-generation sequencing), could significantly contribute to calibrate, improve or even change current models about TB evolution and the spread of MTb complex strains.
Identifying the place of origin and later spread of TB within humans and animals whether wild or under domestication can shed light on its evolutionary process (Lee et al.). A genetic factor is not the only cause for the (re-)emergence of $\mathrm{TB}$, as others must also be considered such as international travel and trade, poor economic environment, demographic (human population increase) and socio-cultural factors (urbanization), a weak health system, together with worldwide human migrations and community of pathogens (Donoghue et al.). Several studies have provided evidence for a role of the host genetics in susceptibility to TB, while numerous candidate genes studies as well as genome-wide linkage scans have been carried out. However, some inconsistencies are noticeable across these studies. In fact, different key elements should be accounted for when conducting forthcoming studies: definition of the controls is clearly questioned (are they true controls or latently infected with tuberculosis?), population substructure (admixture) and differences in $M$. tuberculosis strains (Perrin).

TB is caused by a group of phylogenetically closely related bacteria, known as the M. tuberculosis complex (MTBC). What are the potential evolutionary consequences of such long-standing human-pathogen association? How was TB "seeded" in human populations and when? These questions remain open. Wholemodern genome sequencing, systems biology and host-pathogen interactions are generating new approaches for the understanding of the past and and ongoing evolution, as well as the pathogenicity, of M. tuberculosis. CRISPR (Clustered Regularly Interspersed Short Palindromic Repeats), which demonstrate a historical record of bacterial-phage struggle, have been valuable in the past. Combined to new multi-level SNP-based identification tools, they should remain useful in the future, helping to follow both the microevolution and macroevolution process of MTBC (Sola).

Studies have shown human pathogens to have geographic structures in terms of population genetics. Depending on a splitter/ gatherer point of view, the global population structure of $M$. tuberculosis complex is defined by six to ten phylogeographical lineages, each associated with specific human populations (sympatric). The links between the particular MTBC lineages and human populations are maintained in cosmopolitan settings. Transmission of allopatric strains has been associated with risk factors such as HIV-co-infection. One of the key features of co-evolution is the ongoing evolutionary arms race between the pathogen and the host immune system. Surprisingly, the known human T-cell epitopes of MTBC are the most conserved regions of the MTBC genome and might contribute to the successful spread of MTb. "Ancient" and "modern" lineages can be distinguished among the six main human-adapted lineages (East-African Indian, Beijing, CentralAsian, Euro-American, M. africanum, M. bovis). The three "modern" lineages are more successful in terms of their geographical spread i.e. Europe, China and India. Some studies are consistent with the hypothesis that such strains have evolved a strategy of higher virulence and shorter latency (Mokrousov; Gomes et al.; Simeone et al.). The fight against $M$. tuberculosis also requires better diagnostic platforms (Somoskövi et al.) and new therapeutic approaches (Sipos et al., Horváti et al.). Although the complexity of the co-evolutionary process is difficult to understand, it is key to predicting progressive genetic changes in the future and developing a more integrative view (Perrin).

Questions about the origins and spread of tuberculosis in the past are addressed, in addition to biological and genetic topics, as well as epidemiological problems of the present. A thorough synthesis or concise summary of the articles from such a great number of fields cannot be given here. The emphasis will be different for a paleopathologist and for a microbiologist, just as a clinician and a medical historian approach the evolution of TB in diverse ways. Nevertheless, we remain convinced that the study of TB through an 
interdisciplinary approach as presented in this special issue, including findings from paleopathology to paleogenetics and from modern diversity studies to diagnostics, offers the best potential to significantly improve our knowledge of $\mathrm{TB}$ and its continuing evolution. This special issue represents a small yet significant contribution to the global effort to reduce and hopefully eventually completely eradicate TB infections worldwide. We sincerely believe that by reading this TB Evolution special issue, the general reader will get a comprehensive picture of the issues related to the past and present of Tuberculosis, while the student of its evolution will be equipped with a reliable basis for further research.

December 2014, Szeged-Bordeaux-Montpellier-Paris-Bolzano The Editors

\section{Acknowledgements}

The publication of this special issue titled Tuberculosis in Evolution, dedicated to the past and the evolution of $\mathrm{TB}$, was supported by the University of Szeged, Hungary, the Hungarian Scientific Research Fund 'OTKA' (OTKA Grants No. K78555, K81230, NN
78696), the TÁMOP-4.2.2/B-10/1-2010-0012 project co-financed by the EU and Hungary, the MIVEGEC laboratory (IRD/CNRS) and the University of Montpellier (UM), France, the Université Paris-Sud, France, the Ecole Pratique des Hautes Etudes, France and the Institute for Mummies and the Iceman, EURAC Research, Bolzano, Italy. The Guest Editors are particularly grateful for all these supports.

\section{References}

[1] Pálfi Gy, Dutour O, Deák J, Hutás I, editors. Tuberculosis: past and present. Budapest: TB Foundation, Szeged \& Golden Book Publisher; 1999. p. 608.

[2] WHO. Global tuberculosis report 2014. Geneva: WHO Press, World Health Organization; 2014. ISBN 9789241564809.
György Pálfi Olivier Dutour Pascale Perrin Christophe Sola Albert Zink 